\title{
Cushing Syndrome: Maybe Not So Uncommon of an Endocrine Disease
}

\author{
Federica Guaraldi, MD and Roberto Salvatori, MD
}

Background: Cushing syndrome (CS) is the result of extended exposure to excessive glucocorticoids from endogenous or exogenous sources. Traditionally, the most common cause of endogenous CS is a pituitary adenoma (Cushing disease). Less common causes are adrenocortical tumors and extrapituitary adrenocorticotropin-producing neoplasias.

Objectives: This review provides updated information regarding the potential for increased prevalence of $\mathrm{CS}$ in specific patient populations. Here the authors provide to family physicians clinical guidance for recognition of $\mathrm{CS}$ by presenting a case, discussing the advantages/disadvantages of the diagnostic tests, and discussing information about the treatment options.

Results: CS is expected to have an incidence of 10 to 15 people per million; however, studies of patients with diabetes, obesity, hypertension, and osteoporosis found a high prevalence of CS among these populations. The clinical manifestations of CS range from the distinctive clinical features (purple striae, facial plethora, proximal myopathy) to common conditions such as hypertension, obesity, and diabetes. Clinical practice guidelines recommend biochemical tests to screen patients for CS; however, the sensitivity and specificity of these tests vary, so a careful analysis must be performed to avoid misdiagnosis.

Conclusion: $\mathrm{CS}$ is challenging to diagnose. Nevertheless, with a systematic approach to testing patients and an increased awareness of the high-risk patient populations, the disease can be identified in a timely manner. (J Am Board Fam Med 2012;25:199-208.)

Keywords: Computer-Assisted Diagnosis, Cortisol, Cushing Syndrome, Diagnosis, Metabolic

A 52-year-old, postmenopausal woman had a history of obesity (body mass index $[\mathrm{BMI}]=32$ ), a 4-year history of type 2 diabetes mellitus, and a 3 -year history of hypertension, which were being managed with oral medications. The patient was being treated with metformin and sitagliptin for diabetes (glycosylated hemoglobin [HbAlc] =

This article was externally peer reviewed.

Submitted 22 July 2011; revised 16 September 2011; accepted 23 September 2011.

From the Division of Endocrinology, Department of Medicine, Johns Hopkins University School of Medicine, Baltimore, MD (FG, RS); and the Division of Endocrinology and Metabolism, Department of Internal Medicine, University of Turin, Torino, Italy (FG).

Funding: Financial support for medical editorial assistance was provided by Novartis Pharmaceutical Corporation.

Conflict of interest: Dr Salvatori has received consultant fees from Novartis Pharmaceuticals and from Ipsen/Tercica and is a coinvestigator in clinical trials sponsored by Novartis Pharmaceuticals and Ipsen/Tercica.

Corresponding author: Roberto Salvatori, MD, Pituitary Center, Division of Endocrinology, Johns Hopkins University School of Medicine, 1830 East Monument St, \#333, Baltimore, MD 21287 (E-mail: salvator@jhmi.edu).
$7.8 \%)$, and her hypertension was being controlled with an angiotensin-converting enzyme inhibitor and a calcium channel blocker. In addition, she was being treated with sertraline for depression. The patient referred herself to an urgent care center for persistent foot pain without recollection of trauma. A radiograph revealed a fracture of the third metatarsal bone, which was treated by immobilization. The injury prompted her primary care physician to measure her bone mineral density. The T-score of both the lumbar spine (-2.4) and femoral neck $(-2.3)$ indicated osteopenia. Nevertheless, testing for secondary causes of bone loss were normal (serum calcium, parathyroid hormone, and vitamin D) and the patient was treated with a bisphosphonate to prevent further bone loss.

Four months later, the patient was taken to an emergency department for an episode of acute abdominal pain, diagnosed as diverticulitis. While admitted, a computed tomography (CT) scan of the abdomen detected a low-density adrenal mass measuring $2 \mathrm{~cm}$ on the left side. Follow-up testing 
determined that urine metanephrines were normal, urinary free cortisol (UFC) was just below the upper limit of normal (47 $\mu \mathrm{g} / 24$ hours; upper limit of normal $<50 \mu \mathrm{g} / 24$ hours), and plasma adrenocorticotropic hormone (ACTH) was low $(<5 \mathrm{pg} / \mathrm{mL})$. The diagnosis of Cushing syndrome (CS) was considered and a failure to suppress cortisol $(5.2 \mu \mathrm{g} /$ $\mathrm{dL}$; normal $<1.8 \mu \mathrm{g} / \mathrm{dL}$ ) following a $1-\mathrm{mg}$ dose of dexamethasone (dexamethasone suppression test [DST]), and 2 mildly elevated, late-night salivary cortisol levels confirmed the diagnosis. She underwent a left laparoscopic adrenalectomy. After a 9-month period of adrenal insufficiency, her cortisol secretion normalized. The patient lost weight $(\mathrm{BMI}=271$ year after surgery), her hypertension improved, control of her diabetes was restored $(\mathrm{HbA} 1 \mathrm{C}=6.3 \%)$ while taking metformin only, and she did not experience any new fractures. As the initial contact for patients who demonstrate the signs of cortisol excess, family physician practitioners face the initial challenge of recognizing the signs of hypercortisolism followed by the responsibility of initiating referrals and ensuring the best possible treatment for their patients all while avoiding overtesting their patient.

\section{Hypercortisolism and Cushing Syndrome}

Cushing syndrome is the result of excessive glucocorticoids from either an exogenous or endogenous source. ${ }^{1}$ Exogenous (iatrogenic) CS, the most common form, is caused by excessive oral, intra-articular, topical, or inhaled corticosteroids used as antiinflammatory or immunosuppressive treatments. Because iatrogenic CS is medication dependent, the process to reverse the excessive cortisol exposure is accomplished by tapering the dose with the aim of discontinuing the medication, if possible. Endogenous CS is caused by a disruption to the normal secretory dynamics of the hypothalamuspituitary-adrenal (HPA) axis (Figure 1A), which leads to excessive cortisol secretion. Classically, the most common form of endogenous CS is ACTH dependent and called Cushing disease (CD) when caused by an ACTH-secreting pituitary adenoma, which is thought to occur in $80 \%$ to $85 \%$ of cases (Figure 1B). A small percentage of cases of ACTHdependent CS $(<10 \%)$ is the result of (nonpituitary) ectopic ACTH secretion (EAS) or, more rarely, corticotropin-releasing hormone by benign or malignant neoplasias such as a neuroendocrine tumor (Figure 1D). The remaining percentage of patients (15\% to $20 \%$ ) has ACTH-independent $\mathrm{CS}$, which is the result of either bilateral adrenocortical hyperplasia or adrenocortical tumors that secrete excessive cortisol, causing suppression of ACTH (Figure 1C). The routine use of imaging equipment has increased the identification of incidentally detected adrenal masses. ${ }^{2,3}$ A substantial percentage of these incidentally detected adrenal tumors are hormonally active. ${ }^{2}$ This observation supports the idea that the percentage of patients with ACTH-independent CS is greater than commonly believed.

\section{The Increasing Prevalence of Cushing Syndrome}

There is little epidemiologic information about the incidence and prevalence of CS. It is traditionally estimated to affect 10 to 15 people per million population each year in the United States, which is the reason the Office of Rare Diseases of the National Institutes of Health (NIH) lists it as a "rare disease." 4 Studies conducted in Italy, Spain, and Denmark reported that the annual incidence ranges from 0.7 to 2.4 per million population each year. ${ }^{5-7}$ Although the prevalence in the general population is reported to be a fraction of a percent, recent studies have suggested a much higher prevalence among high-risk patient populations, such as patients with diabetes mellitus (particularly if poorly controlled), hypertension, and early-onset osteoporosis (particularly if with fractures). ${ }^{8-11}$ A study that screened 294 patients with type 2 diabetes mellitus and 189 age-, sex-, and BMI-matched controls by their ability to suppress cortisol determined that the prevalence of subclinical hypercortisolism was higher in diabetic individuals than in controls (9.4\% vs $2.1 \%$, respectively). ${ }^{10}$ Interestingly, the patients' hypercortisolism was primarily from an adrenal origin. Two hundred patients with poorly controlled diabetes mellitus (HbA1C $>8 \%$ ) were screened for hypercortisolism and $5.5 \%$ were diagnosed with CS, mostly of adrenal origin. ${ }^{9}$ In a study of patients with osteoporosis without clinically overt hypercortisolism, $4.8 \%$ of patients (11 of 219) had subclinical hypercortisolism and the prevalence was $10.8 \%$ if they also had a vertebral fracture. ${ }^{11}$ Finally, among 1020 hypertensive patients, $21(2.1 \%)$ had increased levels of cortisol. ${ }^{12}$ These reports will need to be confirmed by larger studies in different regions of the world, and they certainly are affected by the entry selection criteria, but they 
Figure 1. Hypothalamus-pituitary-adrenal (HPA) axis differences between normal patients and patients with Cushing syndrome. A: Normal HPA axis. B: Adrenocorticotropic hormone (ACTH) dependent (Cushing disease). C: ACTH independent (Cushing syndrome). D: Ectopic ACTH syndrome. CRH, corticotropin-releasing hormone.
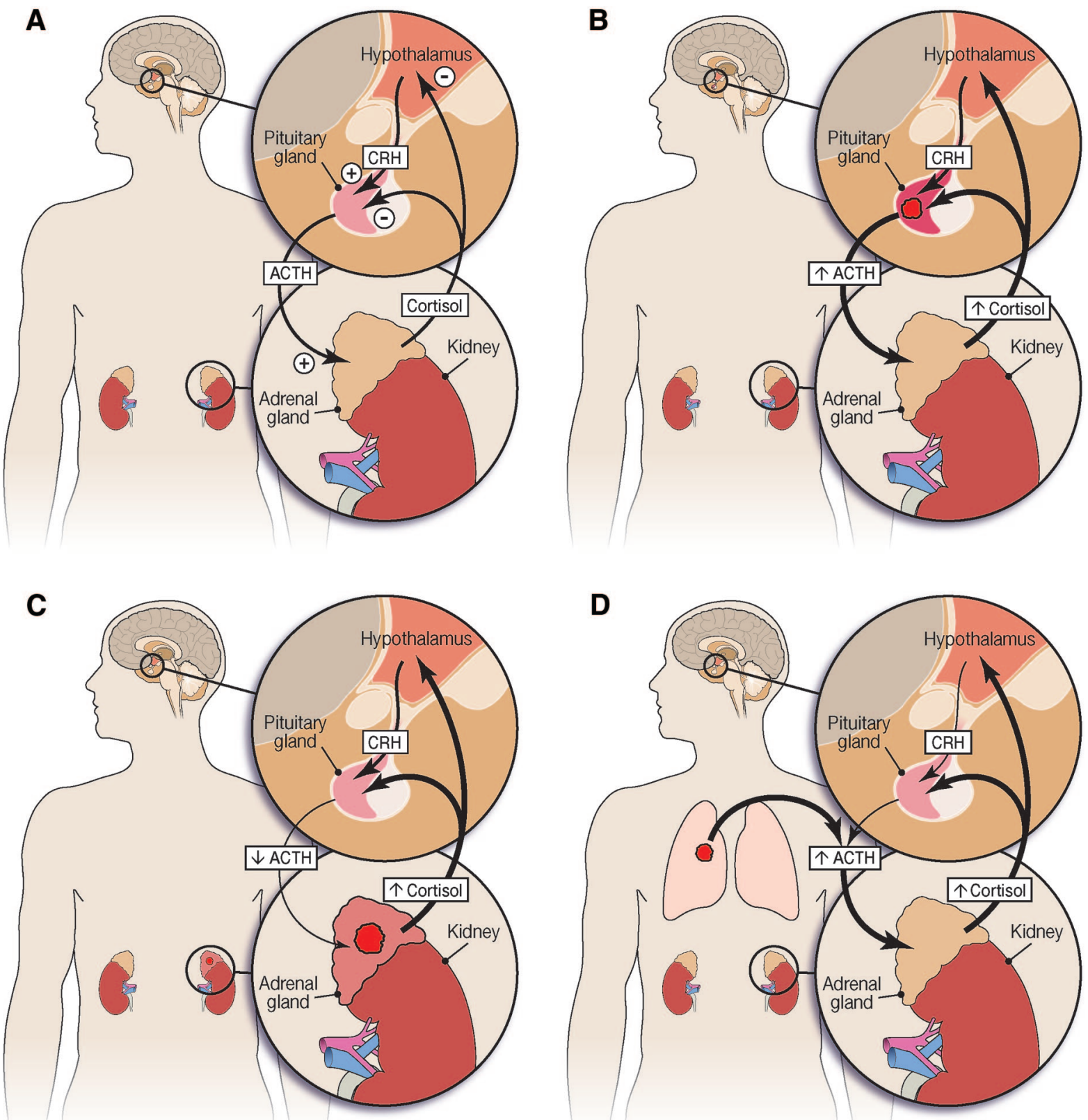

provide a rationale to consider screening for CS even in patients who do not show all the signs of hypercortisolism. These results as a whole lead us to suspect that the real incidence of CS is higher than commonly believed. Nevertheless, the issue of increased screening is controversial, as exemplified in a recent review that argued the drawbacks of screening (cost, acceptability, and unnecessary procedures) may outweigh the benefits. $^{13}$

\section{Recognizing Hypercortisolism in Your Patient}

Early recognition of CS can prevent the long-term physical consequences and increased mortality that may occur when the disease is left untreated. ${ }^{6}$ As illustrated in the patient case study at the beginning of this review, clinical recognition of CS can be complicated and the diagnosis is often delayed. The delay in diagnosis is primarily because the signs and symptoms of hypercortisolism such as obesity, diabetes, hypertension, bone loss, and depression are very com- 
mon among the general population (Table 1). ${ }^{14}$ In addition, the variable degrees to which the signs and symptoms occur require that family physicians are able to recognize both the obvious and subtle signs in their patients and ultimately determine if testing for CS is warranted. Screening for CS should be initiated in all patients with an incidentally discovered adrenal mass, particularly if the CT imaging density is low ( $<20$ Hounsfield units). ${ }^{15}$

As discussed previously, recent studies suggest that CS seems to be more prevalent than was initially thought. These observations not only substantiate the need to test for hypercortisolism in patients who have obvious signs and symptoms of hypercortisolism, but they also pose the question of whether it is necessary to screen patients who have clinical diagnoses that may be caused by endogenous cortisol excess and fall into these high-risk categories. Although there are clinical guidelines that systematically cover the testing methods, diagnosis, and treatment options, at the present time there are no unambiguous guidelines for the family physician that can clearly state which patients to screen and when screening should be performed. The approach "if you are thinking about it, you should do it" may generate a large amount of unnecessary testing, but this obviously would reduce the number of patients who go unrecognized. Ultimately, the difficulty in diagnosing CS is that physicians need to be aware of the signs and symptoms and know the high-risk patient populations for this disease while avoiding excessive low-yield screening that may pose an excessive financial burden on the health system or cause unnecessary worries or surgical procedures.

When the presentation is florid, easily recognizable clinical features of hypercortisolemia include facial plethora, supraclavicular fat pads, buffalo hump, truncal obesity, and purple striae (Table 1). In these patients, biochemical testing can confirm the clinical suspicion. However, when the signs and symptoms are not obvious, "subclinical" (sometimes called "preclinical") CS may be a possibility. This state of disrupted cortisol regulation occurs primarily in ACTHindependent CS. The definition of "subclinical" is preferred because the evolution toward clinically overt hypercortisolism rarely occurs. The criteria for determining subclinical cortisol excess are controversial, and presently there is no consensus definition. An NIH State-of-the-Science Conference concluded that a better term for this condition might be "subclinical autonomous glucocorticoid hypersecretion." 16 The diagnosis requires 2 criteria in addition to some evidence of hypercortisolism such as increased UFC or abnormal DST or salivary test: (1) the patient should not present a clear phenotype of CS, even if some physical stigmata suggestive of hypercortisolism (eg, facial and retrocervical or supraclavicular fullness and central obesity) are present; and (2) the patient should harbor an adrenal mass. With the increasing use of imaging techniques that can identify tumors before clinical symptoms are present, the incidence of subclinical CS cases is increasing and potentially will become much higher than overt CS. ${ }^{2}$ Another diagnostic challenge is the differentiation between mild pituitary CS (ie, CD) and states of hypercortisolism that are seen in chronic stress, depression, and alcohol abuse, referred to as "pseudo-Cushing syndrome." This is a difficult differential diagnosis even in the hands of expert endocrinologists, and therefore this issue will not be discussed in this review.

When clinical suspicion of CS is present, the first step in the diagnosis is to confirm hypercortisolemia. Only after CS has been confirmed should the underlying cause be explored. Advances in the specificity of the biochemical screening tests to measure serum and free cortisol and the sensitivity of imaging methods to identify neoplasms of the pituitary and adrenal glands have both contributed

Table 1. Clinical Features of Cushing Syndrome

\begin{tabular}{|c|c|c|c|}
\hline More Specific Signs & Common Signs & Specific Symptoms & Common Symptoms \\
\hline Striae $(>1 \mathrm{~cm}$ wide $)$ & Acne & None & Depression \\
\hline Facial plethora & Peripheral edema & & Fatigue \\
\hline Proximal myopathy & Muscle weakness & & Weight gain \\
\hline \multirow[t]{4}{*}{ Easy bruising } & Truncal obesity & & Back pain \\
\hline & Supraclavicular fullness & & Irritability \\
\hline & Dorsocervical "buffalo" hump & & Decreased libido \\
\hline & & & Menstrual abnormalities \\
\hline
\end{tabular}


to improvements in recognition of the disease, although there remain many caveats to these techniques. In particular, the high prevalence of pituitary and adrenal "incidentalomas" requires the understanding that the presence of a pituitary or adrenal anatomic abnormality does not necessary mean that they are hormonally active or they require surgical removal. Furthermore, the much higher prevalence of other diseases that share CS symptoms, such as polycystic ovary syndrome, must be always kept in mind.

\section{Biochemical Testing Methods}

The level of cortisol in the body follows a circadian pattern, with the highest levels recorded in the early morning and lowest levels at bedtime. The initial biochemical tests for CS do not need to be completed by an endocrinologist. Family physicians can follow the Endocrine Society's clinical practice guidelines, which recommend one of the following 3 testing methods to screen patients for CS: the 24-hour UFC test, the late-night salivary cortisol test, or the 1-mg overnight DST (Figure 2). ${ }^{14}$ These tests detect elevated cortisol in the urine or saliva or demonstrate abnormal feedback of the HPA axis, all of which characteristic of (but not exclusive to) CS. As mentioned earlier, there are no specific signs exclusive to CS; likewise, there are no exact recommendations describing exactly when to test patients that are suspected of CS. The guidelines recommend using one of the 3 tests for screening "based on its suitability for a given patient." We recommend that family physicians consistently use one test to become comfortable with it and learn its potential pitfalls. Because of the variability of cortisol levels and the suboptimal sensitivity and specificity of the tests, the Endocrine Society recommends at least 2 positive tests before making a diagnosis of CS.

\section{Specific Features of the Biochemical Tests \\ Urinary Free Cortisol}

The UFC test measures a patient's unbound cortisol over a 24-hour period using either antibodybased or high-performance liquid chromatography

Figure 2. Flow chart of the clinical decision process used to diagnose patients suspected of Cushing syndrome (CS). UFC, urinary free cortisol; DST, dexamethasone suppression test; Dex, dexamethasone; CRH, corticotropinreleasing hormone. Copyright $\odot 2009$ Endocrine Society. Reproduced with permission. ${ }^{13}$

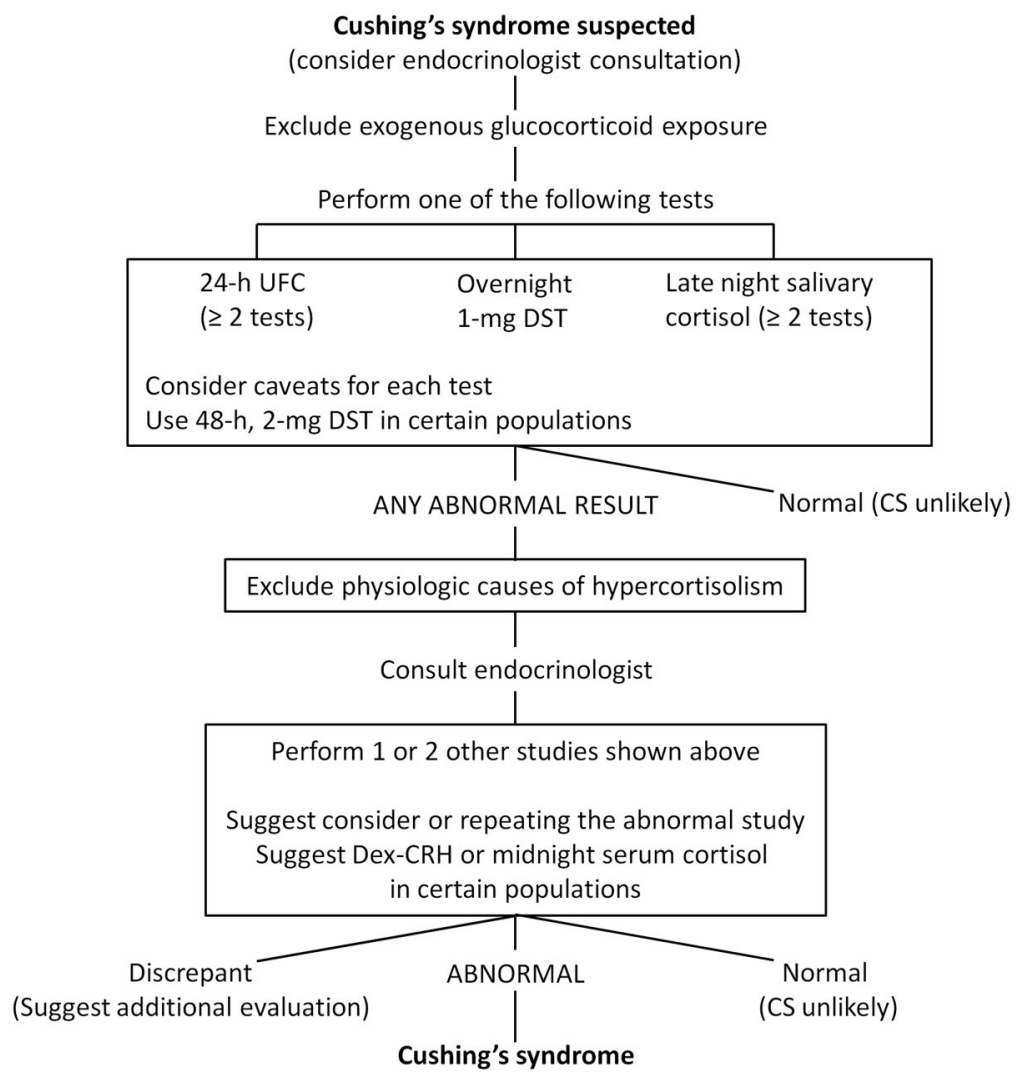


methods. Values greater than 4 times the upper limit of normal are diagnostic of CS, whereas mild hypercortisolism usually falls between the upper limit of normal and 2 to 3 times normal. These excess levels of cortisol are not diagnostic of CS because they may also occur in patients after stressful conditions, depression, or alcohol abuse (ie, pseudo-Cushing syndrome). ${ }^{17}$ Conversely, patients with subclinical CS may have UFC that is within normal limits. To ensure accurate results, the patient's creatinine clearance should be measured because the UFC test is unreliable in patients with creatinine clearance $<60 \mathrm{~mL} / \mathrm{min}^{18}$; moreover, UFC is falsely elevated in patients who produce more than $5 \mathrm{~L}$ of urine a day. ${ }^{19}$ Some medications can have a direct or indirect effect on cortisol levels (eg, digoxin, carbamazepine, and synthetic glucocorticoids) and should also be considered when evaluating the patient's UFC test results. ${ }^{20} \mathrm{In}$ addition to the test results, it is important to confirm that the patient's 24-hour urine volume and urine creatinine are normal because these values may suggest incomplete or excessive collections. Physicians should confirm adherence to the testing protocol because, on inquiry, patients have often admitted that the collection was done improperly. Finally, recalculation of the test should be performed, particularly if the UFC is exceedingly high or low, to confirm that total UFC was correctly calculated from the concentration and volume.

\section{Late-Night Salivary Cortisol}

The late-night salivary cortisol test measures free cortisol levels when its production is at the lowest point in the 24-hour cycle (11 PM to 12 PM in most patients). With CS, patients no longer demonstrate a pattern of cyclic cortisol secretion, and hence the late-night cortisol level no longer reaches a low point. ${ }^{14}$ The collection time should coincide with the patient's normal sleep schedule and should be adjusted in patients who consistently go to bed long after midnight.

This test is now offered by most major commercial laboratories. Patients are given either an empty tube with which to collect saliva, or a cotton tube to chew for 2 to 3 minutes and then place in a plastic tube. Given its high stability at room temperature, the tube can be either transported or mailed to a laboratory for analysis. ${ }^{21}$ Certain patient attributes and habits (eg, irregular sleep schedule, such as in shift workers, or smoking immediately before test- ing) may affect the test results and should be avoided. ${ }^{14,22}$ Also, the effect of blood contamination is not known, so it is best to ask the patient not to brush their teeth immediately before the saliva collection. This testing method is promising for accurately diagnosing CS because it has high diagnostic sensitivity and specificity. ${ }^{23}$ The test correctly identified patients with CS while excluding individuals without the disorder $>90 \%$ of the time $^{23}$; however, recent literature is less supportive of accuracy of this test. ${ }^{24}$

\section{Dexamethasone Suppression Test}

The DST uses the administration of dexamethasone, a synthetic glucocorticoid, to suppress ACTH production and cortisol secretion. The preferred screening test is the overnight 1-mg DST, which measures 8 AM serum cortisol the morning after late-night (11 PM) oral dexamethasone administration. When cortisol levels are less than 1.8 $\mu \mathrm{g} / \mathrm{dL}$, the sensitivity of the overnight DST is $>95 \%$; however, this is at the expense of reduced specificity. ${ }^{25}$ Alternatively, reports comparing the sensitivity of the overnight and 2-day DST suggest that the 2 tests have similar sensitivity. ${ }^{26}$ The patient's history should be reviewed to prevent falsepositive results caused by increased hepatic clearance of dexamethasone from enzyme induction caused by large alcohol consumption or use of drugs such as phenytoin or rifampin. ${ }^{26}$ In ambiguous cases, simultaneous measurement of cortisol and dexamethasone may be performed to ensure that adequate serum dexamethasone levels are reached. Furthermore, increases in serum cortisol due to increased corticosteroid binding globulins caused by oral estrogen may reduce specificity (ie, women taking oral estrogens should not use the DST). ${ }^{27}$

There have been numerous attempts to pinpoint which test has the highest selectivity for diagnosing overt CS, but no one has performed a comparison of the tests in the primary care setting to provide the exact recommendation of who and when to test. A recent meta-analysis determined that the 3 tests have similar accuracy. ${ }^{28}$ When confronted with the task of diagnosing subclinical or mild CS, we find that the salivary cortisol test and DST seem to be more sensitive than the UFC test. Ultimately, in selected cases, biochemical screening tests may not conclusively diagnose CS because they may contradict each other. Given the high incidence of adre- 
nal masses ${ }^{29}$ and pituitary adenomas unrelated to $\mathrm{CS},{ }^{30}$ imaging (magnetic resonance imaging or CT) should only be used after biochemical screening has confirmed the diagnosis of hypercortisolism. In selected cases, the dexamethasone/corticotropin-releasing hormone test is used to differentiate between CD and pseudo-Cushing states, which are situations in which the body produces excessive amounts of cortisol because of ACTH overproduction caused by chronic stress, depression, or alcohol abuse. ${ }^{31,32}$ It is important to understand that none of the tests is $100 \%$ sensitive or specific, and therefore great attention must be paid to avoid misdiagnosis. In our center, we follow a conservative approach and do not proceed to imaging (or surgery) unless we are confident of the biochemical diagnosis. This can cause a great deal of frustration for patients who often want to have CS because they are in search of a curable reason for their ailments, particularly after consulting web sites and chat rooms. We stress the "first do no harm" approach and use repeated testing over long periods of time to establish or exclude the diagnosis of CS ("tincture of time") before surgery.

\section{Identifying the Cause of Cushing Syndrome}

After the diagnosis of CS has been confirmed with biochemical screening tests, the cause of the excess cortisol needs to be determined. ACTH-independent and ACTH-dependent CS are differentiated by measuring the plasma ACTH levels. Patients with ACTH-independent CS usually have suppressed levels of circulating plasma ACTH because of overproduction of cortisol (corticotrophin levels $<5 \mathrm{pg} / \mathrm{mL}$ ), although the degree of suppression may be less marked in subclinical CS. This form of $\mathrm{CS}$, typically caused by adrenal adenomas, can be detected by CT scanning of the adrenals to identify the type and location of the lesion. When plasma ACTH is not suppressed, the diagnosis of ACTHdependent CS is established. Differentiating between CD and EAS (and sometimes between CD and pseudo-Cushing) is sometimes difficult, and the family physician should initiate referral to an endocrinologist who has experience managing patients with CS and access to a tertiary care center for diagnostic testing. Bilateral inferior petrosal sinus sampling is a conclusive invasive technique for discriminating between pituitary and nonpituitary sources of ACTH. In patients with CD, the ACTH levels in the inferior petrosal sinuses will be signif- icantly elevated compared with levels drawn in the periphery. In contrast, in ectopic CS, the ACTH in the inferior petrosal sinuses and the periphery should be similar because the tumor is located elsewhere. ${ }^{33,34}$ It should be stressed that inferior petrosal nasal sampling is not a mean of differentiating Cushing disease from pseudo-Cushing because in both conditions the pituitary is the source of excessive ACTH secretion.

\section{Management and Treatment of Cushing Syndrome Surgical Methods}

The goals for treatment of patients with CS are reversal of clinical features, normalization of cortisol levels, and long-term control without recurrence. ${ }^{35}$ In the presence of ACTH-independent CS caused by an identifiable cortisol-producing adrenal adenoma, the patient should be treated by unilateral adrenalectomy. The benefits of operating on patients with subclinical CS are less obvious, although recent literature demonstrates an advantage in surgery versus observation in weight, blood pressure control, lipid profile, and glucose levels. ${ }^{36-38}$ In cases of bilateral hyperplasia, bilateral adrenalectomy may be an option; however, this treatment will result in adrenal insufficiency and require lifetime hormone replacement therapy with glucocorticoids and mineralocorticoids. Recent literature obtained from a small series of patients points to the potential utility of unilateral adrenalectomy, even in bilateral macronodular hyperplasia, in reducing urinary cortisol, blood pressure, and weight. $16,39,40$ Transsphenoidal adenomectomy (TSA) is the initial treatment of choice for most patients with CD. The goal of TSA is the complete resection of the pituitary adenoma and correction of hypercortisolism without inducing permanent pituitary deficiencies. When performed by an experienced pituitary surgeon on an identified microor macroadenoma, TSA produces the fastest cure. Initial remission rates after TSA for patients with microadenomas are variable $(65 \%$ to $90 \%)^{35}$ and depend on surgical skill as well as tumor size and location. ${ }^{41,42}$ Repeat pituitary surgery and pituitary radiotherapy are sometimes used for patients with persistent hypercortisolism after initial TSA. However, repeat pituitary surgery carries with it the significant risk of pituitary insufficiency and is recommended only when evidence of a remaining pituitary adenoma is established. Conventional radiotherapy and targeted 
radiosurgery ( $\gamma$-knife or cyberknife) have the potential to eradicate the pituitary tumor, but control of hypercortisolemia occurs in approximately $50 \%$ to $60 \%$ of patients within 3 to 5 years, ${ }^{35}$ and significant pituitary deficiency may develop after treatment.

\section{Medical Therapy}

Of the traditional approaches described, each has notable drawbacks that can lead to lasting hormone deficiency or mortality. ${ }^{36}$ Therefore, continued development of new therapies that target the underlying cause of hypercortisolism is needed. In patients with CS who are not candidates for surgery or have not achieved remission after initial surgery, there are various options for medical treatment. Adrenal-directed therapies with steroidogenesis inhibitors, such as metyrapone (not routinely available in the United States) and ketoconazole, are used to directly inhibit the secretion of cortisol by the adrenal gland. These are palliative treatments because they do not treat the underlying cause or restore normal HPA secretory dynamics. ${ }^{20}$ Furthermore, ketoconazole may cause liver damage and requires an acidic $\mathrm{pH}$ to be absorbed. Preliminary results following the treatment of patients with CS using the glucocorticoid receptor antagonist mifepristone (RU486; Corcept Therapeutics, Menlo Park, CA) were recently presented at the 2011 Endocrine Society meeting. The SEISMIC study demonstrated restoration of glucose tolerance in $60 \%$ of glucose-intolerant patients or lowering of diastolic blood pressure in $43 \%$ of hypertensive patients. ${ }^{43}$ However, the therapy is difficult to manage because it does not reduce cortisol levels, and therefore the physician has no biochemical assay to use to adjust dosing. For patients with CD, a pituitary-directed medical treatment to normalize ACTH secretion, restore cortisol levels, and inhibit tumor growth would represent a major advance. Initial promising results with the dopaminergic agent cabergoline (approved for treatment of prolactinomas) were followed by data showing frequent escape from the effect of the drug on cortisol levels. ${ }^{44}$ Results from a 12-month phase III study with a new somatostatin analog, pasireotide (SOM230; Novartis, East Hanover, NJ), were recently presented at the 2011 Endocrine Society meeting. The study reported a decrease in UFC by 6 months in the majority of patients and normalization of UFC in $25 \%$ of patients with CD after 12 months. ${ }^{45}$

\section{Conclusions}

Recognition of CS and the identification of the underlying cause are often challenging. Even more challenging is the decision about whom to screen in the primary care setting. To date, evidence-based guidelines for screening criteria are missing. The Endocrine Society clinical practice guidelines recommend testing for CS in patients with multiple signs and symptoms compatible with the syndrome. In addition to the treatment guidelines, patients in high-risk groups such as those with poorly controlled diabetes, hypertension, and early-onset osteoporosis (particularly with fractures) have been shown to have a high prevalence of subclinical (mostly ACTH-independent) CS. The issue of whether it is cost-effective to screen these groups of patients is still unresolved. Testing should be done in patients who are incidentally discovered to have an adrenal mass. Only a small percentage of these will turn out to have CS. Efficient biochemical screening tests represent the most valuable tool to diagnose the disease. Once diagnosed, the first-line treatment strategy is surgery to remove the tumor. However, because surgery is not effective in all patients with $\mathrm{CD}$, additional treatment is needed and can include repeat surgery, radiation therapy, and medical therapy.

The authors would like to thank Timothy Remus, $\mathrm{PhD}$, for medical editorial assistance in the preparation of this manuscript.

\section{References}

1. Arnaldi G, Angeli A, Atkinson AB, et al. Diagnosis and complications of Cushing syndrome: a consensus statement. J Clin Endocrinol Metab 2003;88:5593-602.

2. Reincke M. Subclinical Cushing syndrome. Endocrinol Metab Clin North Am 2000;29:43-56.

3. Kloos RT, Gross MD, Francis IR, Korobkin M, Shapiro B. Incidentally discovered adrenal masses. Endocr Rev 1995;16:460-84.

4. National Institutes of Health, Office of Rare Diseases Research. Rare Diseases and Related Terms. http://rarediseases.info.nih.gov/RareDiseaseList.aspx? PageID =1. Accessed January 25, 2012.

5. Ambrosi B, Faglia G; Multicenter Pituitary Tumor Study Group. Epidemiology of pituitary tumors. In: Faglia G, Beck-Peccoz P, Ambrosi B, Travaglini P, Spada A (eds.). Pituitary Adenomas: New Trends in Basic and Clinical Research. Amsterdam: Excerpta Medica; 1991:159-68.

6. Etxabe J, Vazquez JA. Morbidity and mortality in Cushing disease: an epidemiological approach. Clin Endocrinol (Oxf) 1994;40:479-84. 
7. Lindholm J, Juul S, Jorgensen JO, et al. Incidence and late prognosis of Cushing syndrome: a population-based study. J Clin Endocrinol Metab 2001;86: 117-23.

8. Anderson GH Jr, Blakeman N, Streeten DH. The effect of age on prevalence of secondary forms of hypertension in 4429 consecutively referred patients. J Hypertens 1994;12:609-15.

9. Catargi B, Rigalleau V, Poussin A, et al. Occult Cushing syndrome in type-2 diabetes. J Clin Endocrinol Metab 2003;88:5808-13.

10. Chiodini I, Torlontano M, Scillitani A, et al. Association of subclinical hypercortisolism with type 2 diabetes mellitus: a case-control study in hospitalized patients. Eur J Endocrinol 2005;153:837-44.

11. Chiodini I, Mascia ML, Muscarella S, et al. Subclinical hypercortisolism among outpatients referred for osteoporosis. Ann Intern Med 2007;147:541-8.

12. Omura M, Saito J, Yamaguchi K, Kakuta Y, Nishikawa T. Prospective study on the prevalence of secondary hypertension among hypertensive patients visiting a general outpatient clinic in Japan. Hypertens Res 2004;27:193-202.

13. Tabarin A, Perez P. Pros and cons of screening for occult Cushing syndrome. Nat Rev Endocrinol 2011;7:445-55.

14. Nieman LK, Biller BM, Findling JW, et al. The diagnosis of Cushing syndrome: an Endocrine Society Clinical Practice Guideline. J Clin Endocrinol Metab 2008;93:1526-40.

15. Hamrahian AH, Ioachimescu AG, Remer EM, et al. Clinical utility of noncontrast computed tomography attenuation value (hounsfield units) to differentiate adrenal adenomas/hyperplasias from nonadenomas: Cleveland Clinic experience. J Clin Endocrinol Metab 2005;90:871-7.

16. Grumbach MM, Biller BM, Braunstein GD, et al. Management of the clinically inapparent adrenal mass ("incidentaloma"). Ann Intern Med 2003;138:424-9.

17. Yanovski JA, Cutler GB Jr. Glucocorticoid action and the clinical features of Cushing syndrome. Endocrinol Metab Clin North Am 1994;23:487-509.

18. Chan KC, Lit LC, Law EL, et al. Diminished urinary free cortisol excretion in patients with moderate and severe renal impairment. Clin Chem 2004;50:757-9.

19. Mericq MV, Cutler GB Jr. High fluid intake increases urine free cortisol excretion in normal subjects. J Clin Endocrinol Metab 1998;83:682-4.

20. Newell-Price J, Bertagna X, Grossman AB, Nieman LK. Cushing syndrome. Lancet 2006;367:1605-17.

21. Garde AH, Hansem AM. Long-term stability of salivary cortisol Scan J Clin Lab Invest 2005;65(5): 433-6.

22. Badrick E, Kirschbaum C, Kumari M. The relationship between smoking status and cortisol secretion. J Clin Endocrinol Metab 2007;92:819-24.

23. Papanicolaou DA, Mullen N, Kyrou I, Nieman LK. Nighttime salivary cortisol: a useful test for the di- agnosis of Cushing syndrome. J Clin Endocrinol Metab 2002;87:4515-21.

24. Baid SK, Sinaii N, Wade M, Rubino D, Nieman LK. Radioimmunoassay and tandem mass spectrometry measurement of bedtime salivary cortisol levels: a comparison of assays to establish hypercortisolism. J Clin Endocrinol Metab 2007;92:3102-7.

25. Wood PJ, Barth JH, Freedman DB, Perry L, Sheridan B. Evidence for the low dose dexamethasone suppression test to screen for Cushing syndrome: recommendations for a protocol for biochemistry laboratories. Ann Clin Biochem 1997;34:222-9.

26. Putignano P, Kaltsas GA, Satta MA, Grossman AB. The effects of anti-convulsant drugs on adrenal function. Horm Metab Res 1998;30:389-97.

27. Nickelsen T, Lissner W, Schöffling K. The dexamethasone suppression test and long-term contraceptive treatment: measurement of ACTH or salivary cortisol does not improve the reliability of the test. Exp Clin Endocrinol 1989;94:275-80.

28. Elamin MB, Murad MH, Mullan R, et al. Accuracy of diagnostic tests for Cushing syndrome: a systematic review and metaanalyses. J Clin Endocrinol Metab 2008;93:1553-62.

29. Mantero F, Terzolo M, Arnaldi G, et al. A survey on adrenal incidentaloma in Italy. Study Group on Adrenal Tumors of the Italian Society of Endocrinology. J Clin Endocrinol Metab 2000;85:637-44.

30. Ezzat S, Asa SL, Couldwell WT, et al. The prevalence of pituitary adenomas: a systematic review. Cancer 2004;101:613-9.

31. Nieman LK, Oldfield EH, Wesley R, Chrousos GP, Loriaux DL, Cutler GB Jr. A simplified morning ovine corticotropin-releasing hormone stimulation test for the differential diagnosis of adrenocorticotropin-dependent Cushing syndrome. J Clin Endocrinol Metab 1993;77:1308-12.

32. Reimondo G, Paccotti P, Minetto M, et al. The corticotrophin-releasing hormone test is the most reliable noninvasive method to differentiate pituitary from ectopic ACTH secretion in Cushing syndrome. Clin Endocrinol (Oxf) 2003;58:718-24.

33. Lindsay JR, Nieman LK. Differential diagnosis and imaging in Cushing syndrome. Endocrinol Metab Clin North Am 2005;34:403-21.

34. Oldfield EH, Doppman JL, Nieman LK, et al. Petrosal sinus sampling with and without corticotropin-releasing hormone for the differential diagnosis of Cushing syndrome. N Engl J Med 1991;325:897905.

35. Biller BM, Grossman AB, Stewart PM, et al. Treatment of adrenocorticotropin-dependent Cushing syndrome: a consensus statement. J Clin Endocrinol Metab 2008;93:2454-62.

36. Guerrieri M, Campagnacci R, Patrizi A, Romiti C, Arnaldi G, Boscaro M. Primary adrenal hypercortisolism: minimally invasive surgical treatment or medical therapy? A retrospective study with long- 
term follow-up evaluation. Surg Endosc 2010;24: 2542-6.

37. Toniato A, Merante-Boschin I, Opocher G, Pelizzo MR, Schiavi F, Ballotta E. Surgical versus conservative management for subclinical Cushing syndrome in adrenal incidentalomas: a prospective randomized study. Ann Surg 2009;249:388-91.

38. Chiodini I, Morelli V, Salcuni AS, et al. Beneficial metabolic effects of prompt surgical treatment in patients with an adrenal incidentaloma causing biochemical hypercortisolism. J Clin Endocrinol Metab 2010;95:2736-45.

39. Iacobone M, Albiger N, Scaroni C, et al. The role of unilateral adrenalectomy in ACTH-independent macronodular adrenal hyperplasia (AIMAH). World J Surg 2008;32:882-9.

40. Mazzuco TL, Bourdeau I, Lacroix A. Adrenal incidentalomas and subclinical Cushing syndrome: diagnosis and treatment. Curr Opin Endocrinol Diabetes Obes 2009;16:203-10.

41. Hammer GD, Tyrrell JB, Lamborn KR, et al. Transsphenoidal microsurgery for Cushing disease: initial outcome and long-term results. J Clin Endocrinol Metab 2004;89:6348-57.

42. Rees DA, Hanna FW, Davies JS, Mills RG, Vafidis J, Scanlon MF. Long-term follow-up results of transsphenoidal surgery for Cushing disease in a single centre using strict criteria for remission. Clin Endocrinol (Oxf) 2002;56:541-51.

43. Fleseriu M, Biller BM, Findling J, et al. Mifepristone, a glucocorticoid receptor antagonist, produces clinical and metabolic benefits in patients with refractory cushing syndrome: results from the Study of the Efficacy and Safety of Mifepristone in the Treatment of Endogenous Cushing Syndrome (SEISMIC). Endocr Rev 2011;32:OR09-5.

44. Petrossians P, Thonnard A-S, Beckers A. Medical treatment in Cushing syndrome: dopamine agonists and cabergoline. Neuroendocrinology 2010; 92(Suppl 1):116-9.

45. Colao A, Petersenn S, Newell-Price J, et al. Pasireotide (SOM230) demonstrates efficacy in patients with Cushing disease: results from a large, randomized-dose, double-blind, Phase III Study. Endocr Rev 2011;32:abst OR09-6. 